\section{Associação entre o índice de massa corporal de pais e de escolares de 7 a 14 anos de Florianópolis, SC, Brasil}

Carla de Oliveira Bernardo 1

Patrícia Salvador Fernandes 2

Rosângela Mirela Marchi Beltrão Campos 3

Fernando Adami 4

Francisco de Assis Guedes de Vasconcelos 5

\section{The association between the Body Mass Index of schoolchildren aged between 7 and 14 years and that of their parents in the city of Florianópolis, in the State of Santa Catarina, Brazil}

1-3,5 Departamento de Nutrição. Centro de Ciências da Saúde. Universidade Federal de Santa Catarina. Campus Universitário Trindade. Florianópolis, SC, Brasil. CEP 88.040-900. E-mail: carlabernardoo@gmail.com

4 Programa de Pós-graduação em Saúde Pública. Universidade de São Paulo. São Paulo, SP, Brasil.

\begin{abstract}
Objectives: to assess the association between parents' Body Mass Index and schoolchildren from the age of 7 to 14 years old.

Methods: cross sectional epidemiological study with 886 schoolchildren from two public and two private schools in Florianópolis Island, Santa Catarina state. Schoolchildren and theirs parents anthropometric diagnostic was defined from the respectively BMI for age according to the Centers for Disease Control and Prevention and WHO cutoff points. The association between parents BMI and schoolchildren was estimated through the prevalence ratio (PR) with confidence interval (CI) 95\% and Pearson's chi-square test with the significant value of $p<0.05$.
\end{abstract}

Results: higher overweight and obesity prevalence was verified in male students, $29.9 \%$, compare to $17.7 \%$ to female students $(p<0.001)$. Significant statistical relation between female students BMI and theirs mothers BMI was found, according to $P R=1.63 ; C I 95 \%=1.1-3.0 ; p=0.02$ and theirs fathers $B M I, P R=1.78 ; C I 95 \%=1.1-3.5 ; p=0.01$. The association between male students IMC and theirs mothers and fathers did not show significant statistical relation.

Conclusions: the female students prevalence of show overweight or obesity is 1.63 times higher when theirs mothers also showed the same nutritional status and 1.78 times higher when the father shows overweight/obesity in comparison to mothers and fathers with normal weight or underweight.

Key words Body mass index, Obesity, Students, Parents

\section{Resumo}

Objetivos: verificar a associação entre o Índice de Massa Corporal (IMC) de escolares de 7 a 14 anos e dos respectivos pais.

Métodos: estudo transversal com 886 escolares de quatro escolas de Florianópolis, SC. Diagnóstico antropométrico dos escolares e dos pais definido, respectivamente, a partir do IMC para idade de acordo com Centers for Disease Control and Prevention e dos pontos de corte da Organização Mundial da Saúde. A associação entre o IMC dos pais e dos escolares foi estimada por meio da razão de prevalência (RP) com intervalo de confiança (IC) de 95\% e teste qui-quadrado com valor de significância de $p<0,05$.

Resultados: identificou-se prevalência de sobrepeso/obesidade mais elevada em meninos $(29,9 \%)$ quando comparada a de meninas $(17,7 \%)$ $(p<0,001)$. Observou-se relação estatisticamente significante entre o IMC de escolares do sexo feminino com o IMC das mães ( $R P=1,63 ;$ IC95\%=1,1$3,0 ; p=0,02)$ e dos pais $(R P=1,78 ; I C 95 \%=1,1-3,5$; $p=0,01)$. Nos escolares do sexo masculino a associação observada não foi estatisticamente significante.

Conclusões: identificou-se que a prevalência do sobrepeso ou obesidade é 1,63 vezes maior, entre as meninas, quando a mãe também apresenta esse distúrbio e 1,78 vezes maior quando o pai o apresenta, em comparação a mães e pais eutróficos ou de baixo peso.

Palavras-chave Índice de massa corporal, Obesidade, Estudantes, Pais 


\section{Introdução}

A obesidade na infância e adolescência é uma relevante questão de saúde pública devido ao rápido aumento de sua prevalência em diversos países do mundo e suas consequências médico-sociais agregadas.1,2 Ela é responsável pelo aumento da morbimortalidade na população, sendo associada a outras doenças crônicas não transmissíveis como diabetes, dislipidemias e hipertensão arterial. ${ }^{3}$

Um estudo sobre a tendência da obesidade em crianças e adolescentes mostrou que a prevalência de sobrepeso no Brasil triplicou de 4,1\% para 13,9\% entre os anos de 1974 e 1997.4 Dados mais recentes, originados pela Pesquisa de Orçamento Familiar realizada em 2002 pelo Instituto Brasileiro de Geografia e Estatística (IBGE) atestam que a prevalência de sobrepeso, incluindo obesidade em meninos e meninas de 10 a 19 anos, é de $17,9 \%$ e $15,4 \%$, respectivamente. 5 Nesse mesmo ano, uma pesquisa realizada no município de Florianópolis com o objetivo de avaliar o estado antropométrico de escolares de 7 a 10 anos de ambos os sexos, com base no índice de massa corporal (IMC), encontrou prevalência de obesidade de $5,5 \%$ e de sobrepeso incluindo obesidade de $22,1 \%{ }^{6}$

Whitaker et al. 7 relatam a necessidade da identificação precoce do excesso de peso em crianças para diminuir o risco de se tornarem adultos obesos. Acerca disso, observa-se que a infância e adolescência são períodos críticos para o início da obesidade e apesar de ainda não se conhecer claramente sua etiologia, sabe-se que muitos são os fatores de risco envolvidos na sua gênese,, 8 sendo um dos mais importantes a presença de obesidade nos pais, representando a correlação da influência genética e ambiental. ${ }^{9}$

Esses achados corroboram com os encontrados por Engstron e Anjos, ${ }^{10}$ a partir dos dados da Pesquisa Nacional sobre Saúde e Nutrição com crianças menores de 10 anos, que mostraram que o estado antropométrico das mães e filhos brasileiros tem grande correlação quando se trata de sobrepeso. Os resultados indicaram que filhos de mães com sobrepeso apresentavam 3,19 vezes mais risco de também desenvolverem sobrepeso em comparação às mães com baixo peso e 2,46 vezes mais chance em comparação às mães com estado nutricional adequado.

No ano de 2000, em Brasília, em estudo desenvolvido entre escolares de ambos os sexos com idades de seis a dez anos, observou-se que a frequência de sobrepeso e obesidade foi de $51,8 \%$, $50 \%$ e $19,6 \%$ respectivamente, na presença de obesi- dade em ambos os pais, em apenas um deles e quando nenhum era obeso. Os resultados mostraram, portanto, correlação significante entre o sobrepeso e obesidade dos pais e dos filhos $(p<0,001){ }^{11}$

Da mesma maneira, na pesquisa desenvolvida por Guimarães et al.12 com escolares de 6 a 11 anos de idade da cidade de Cuiabá, o IMC dos familiares, principalmente o da mãe, mostrou associação estatística significante com o sobrepeso das crianças (IMC $\geq$ percentil 85 ), associação também identificada na análise de regressão logística múltipla.

Com base no exposto, o objetivo do presente estudo foi verificar a associação entre o IMC de pais e de seus filhos escolares de 7 a 14 anos no município de Florianópolis, SC.

\section{Métodos}

Trata-se de um estudo de corte transversal, subprojeto de uma pesquisa mais ampla (Conselho Nacional de Desenvolvimento Científico e Tecnológico, Processo n ${ }^{\circ} 402322 / 2005-3$ ) que teve como objetivo o monitoramento da evolução da composição corporal, tendência e prevalência de sobrepeso, obesidade e baixo peso e sua relação com o estilo de vida em escolares de 7 a 14 anos de idade do município de Florianópolis, SC, nos anos de 2002 e 2007, onde foram utilizados apenas os dados coletados no ano de 2007.

Para o cálculo do tamanho da amostra considerou-se $10 \%$ de prevalência de obesidade entre escolares de 7 a 10 anos de idade 13 e de $17 \%$ para os escolares de 11 a 14 anos de idade, 14 com $95 \%$ de confiança e $2 \%$ de erro amostral (bicaudal). O efeito do desenho é de 1,3 e o poder de $80 \% .15$ Foi acrescentado um valor de $10 \%$ para possíveis perdas na amostra.

Para a estimativa da população escolar na faixa etária de 7 a 14 anos foram utilizadas as informações do censo escolar de 2004 em Santa Catarina, com um universo de 54 mil alunos, distribuídos nas escolas no município de Florianópolis. Para esse subprojeto foi selecionada uma subamostra com o total de alunos pesquisados em quatro escolas (duas públicas e duas particulares). Essas escolas encontram-se na região central, com concentração de 50\% de todos os alunos do município. A escolha dos alunos foi feita de forma aleatória. Os critérios de inclusão foram: ter idade entre 7 e 14 anos, ter consentimento informado autorizado pelos pais ou responsáveis e querer participar estudo.

Dos 903 escolares selecionados 17 foram excluídos da amostra por recusa à pesagem, sendo válidos os dados de 886 participantes. Entre os 
familiares dos 886 escolares, participaram da pesquisa $831(93,8 \%)$ mães e $737(83,2 \%)$ pais, o que representa uma perda de $6,2 \%$ e $16,8 \%$, respectivamente. Foram excluídos: 54 mães e 147 pais por ausência do preenchimento dos dados de peso e estatura no questionário e uma mãe e dois pais por apresentarem valores de medidas antropométricas acima do limite estabelecido, de \pm 4 desvios-padrão em relação à média da população de referência. 16

Os entrevistadores foram treinados em dois workshops e realizou-se um estudo piloto em uma escola diferente das amostradas para ajustar a logística da investigação.

A coleta das medidas antropométricas dos escolares seguiu os procedimentos recomendados por Lohman et al. 17 e pela World Health Organization (WHO). 18 Foi aplicado um questionário socioeconômico aos pais considerando as variáveis: idade, sexo, peso, estatura e rede de ensino (pública ou particular) dos escolares, entre os meses de março e julho de 2007.

A pesagem dos escolares foi obtida em quilogramas, utilizando-se balança digital com capacidade de até $180 \mathrm{~kg}$ e definição de 100 gramas, realizada com os participantes usando roupas leves, descalços e posicionados no centro da plataforma da balança.

Foi utilizado um estadiômetro (precisão de $1 \mathrm{~mm}$ ), para a medição da estatura (em centímetros) realizada com os escolares sem sapatos, em posição ortostática, com o peso distribuído em ambas as pernas, braços ao longo do corpo, pés unidos, joelhos esticados e a cabeça orientada no plano horizontal de Frankfurt (plano para a orientação cefálica, no qual o indivíduo permanece com o olhar no horizonte, sendo que uma linha imaginária passa na cabeça, tangendo a borda superior dos condutos auditivos externos e o ponto mais baixo na margem da órbita ocular). ${ }^{17}$ A medida foi obtida em apnéia após uma inspiração profunda, para promover a descompressão da coluna vertebral.

O diagnóstico antropométrico dos escolares foi definido a partir do IMC para idade de acordo com dados de referência do Centers for Disease Control and Prevention (CDC). ${ }^{19}$ Foram consideradas com baixo peso as crianças com IMC $\leq$ percentil 5 ; eutróficas com IMC entre os percentis 5 e 85; com sobrepeso as crianças com IMC $\geq$ percentil 85 e obesas com IMC $\geq$ percentil 95.

Foram utilizadas para os pais medidas antropométricas autorreferidas, coletadas no momento do preenchimento do questionário. Essa técnica de medição tem sido utilizada em estudos internacionais 20,21 e nacionais, 22,23 sendo mais utilizada em estudos envolvendo um grande número de indivíduos, favorecendo a economia de recursos e a simplificação no trabalho de campo, ${ }^{24}$ e mostram uma fidedignidade adequada das medidas. Neste estudo, foram considerados com baixo peso os adultos com IMC $<18,5 \mathrm{~kg} / \mathrm{m}^{2}$; eutróficos, com IMC entre 18,5 e $24,9 \mathrm{~kg} / \mathrm{m}^{2}$; com sobrepeso aqueles com IMC entre 25 e $29,9 \mathrm{~kg} / \mathrm{m}^{2}$ e obesos com IMC $\geq$ $30 \mathrm{~kg} / \mathrm{m}^{2}$, de acordo com a WHO. 18

Para a realização das análises os escolares e seus pais foram classificados em dois grupos de acordo com a presença ou ausência de sobrepeso/obesidade: escolares com sobrepeso/obesidade (IMC $\geq$ percentil 85) e sem sobrepeso/obesidade (IMC $<$ percentil 85); pais com sobrepeso/obesidade (IMC $\geq 25)$ e sem sobrepeso/obesidade (IMC $<25$ ).

Os dados foram processados e analisados no software Statistical Package for Social Sciences (SPSS) versão 10.0 for Windows. Na análise estatística considerou-se o efeito do desenho de 1,3. Foi realizada análise descritiva dos dados, a partir do cálculo de medidas de tendência central e de dispersão (média, mediana, desvio-padrão, valores mínimos e máximos) e da prevalência do estado antropométrico por sexo, faixa etária e rede de ensino. A associação entre o IMC dos pais e dos escolares foi estimada por meio da razão de prevalência com intervalo de confiança (IC) de 95\% e do teste qui-quadrado com valor de significância de $p<0,05$. A razão de prevalência é obtida pela divisão da prevalência do desfecho (sobrepeso e obesidade nos escolares) entre o grupo de expostos (pai/mãe com sobrepeso/obesidade) e não expostos (pai/mãe sem sobrepeso/obesidade). 25

O protocolo da pesquisa foi aprovado pelo Comitê de Ética da Pesquisa com Seres Humanos da Universidade Federal de Santa Catarina/CCS em 24 de abril de 2006 (parecer 028/06). O Termo de Consentimento Livre e Esclarecido assinado pelos pais dos alunos consistiu no principal instrumento para autorização da participação dos escolares no estudo.

\section{Resultados}

Participaram do estudo 886 escolares de 1a. à 8 a. série do ensino fundamental: $561(63,3 \%)$ são das escolas públicas, sendo $238(42,4 \%)$ do sexo masculino e $323(57,6 \%)$ do sexo feminino; 325 $(36,7 \%)$ são das escolas particulares, com 156 (48\%) do sexo masculino e 169 (52\%) do sexo feminino. Entre os familiares, participaram da pesquisa 831 mães e 737 pais já referidos.

Os valores de medidas de tendência central e de 
Tabela 1

Distribuição dos valores de medidas de tendência central e de dispersão para as variáveis antropométricas dos escolares de 7 a 14 anos e dos seus pais do município de Florianópolis, SC, 2007.

\begin{tabular}{|c|c|c|c|c|c|c|}
\hline Variáveis & $\mathbf{N}$ & $\bar{x} \pm D P$ & Mediana & \multicolumn{3}{|c|}{ Mínimo - Máximo } \\
\hline \multicolumn{7}{|l|}{ Escolares } \\
\hline Idade (anos) & 886 & $11,6 \pm 2,2$ & 12,1 & 7,0 & - & 14,0 \\
\hline Peso $(\mathrm{kg})$ & 886 & $45,3 \pm 14,7$ & 44,3 & 21,0 & - & 87,0 \\
\hline Estatura $(\mathrm{cm})$ & 886 & $150,4 \pm 15,7$ & 151,4 & 116,8 & - & 189,0 \\
\hline $\mathrm{IMC}\left(\mathrm{kg} / \mathrm{m}^{2}\right)$ & 886 & $19,5 \pm 3,5$ & 18,9 & 13,1 & - & 32,4 \\
\hline \multicolumn{7}{|l|}{ Pais } \\
\hline Peso (kg) & 741 & $79,9 \pm 13,1$ & 79,0 & 45,0 & - & 140,0 \\
\hline Estatura $(\mathrm{cm})$ & 775 & $174,6 \pm 7,7$ & 175,0 & 140,0 & - & 198,0 \\
\hline IMC (kg/m²) & 737 & $26,1 \pm 3,7$ & 25,7 & 15,5 & - & 45,9 \\
\hline \multicolumn{7}{|l|}{ Mães } \\
\hline Peso $(\mathrm{kg})$ & 836 & $63,8 \pm 10,9$ & 62,0 & 40,0 & - & 116,0 \\
\hline Estatura $(\mathrm{cm})$ & 840 & $162,7 \pm 6,6$ & 163,0 & 130,0 & - & 185,0 \\
\hline IMC $\left(\mathrm{kg} / \mathrm{m}^{2}\right)$ & 831 & $24 \pm 3,9$ & 23,2 & 16,2 & - & 44,7 \\
\hline
\end{tabular}

IMC=índice de massa corporal.

dispersão para as variáveis antropométricas dos escolares e de seus pais são apresentados na Tabela 1.

As prevalências de baixo peso, sobrepeso e obesidade entre os escolares do sexo masculino foram de $3,3 \%, 17,3 \%$ e $12,7 \%$, respectivamente; no sexo feminino, foram $4,5 \%$ de baixo peso, $10,6 \%$ de sobrepeso e 7,1\% de obesidade; a prevalência de sobrepeso incluindo obesidade foi de $29,9 \%$ no sexo masculino e $17,8 \%$ no sexo feminino, com diferença estatisticamente significante segundo o sexo $(p<0,001)$.

A análise das variáveis que expressam o estado antropométrico indicou prevalência de sobrepeso/ obesidade maior entre as meninas da rede pública de ensino $(20,4 \%)$ do que entre aquelas da rede particular $(12,4 \%)(p=0,027)$. Não houve significância estatística entre os escolares do sexo masculino (Tabela 2)

\section{Tabela 2}

Distribuição absoluta e percentual de escolares, pais e mães com sobrepeso/obesidade segundo tipo de escola. Florianópolis, SC, 2007.

\begin{tabular}{|c|c|c|c|c|c|}
\hline & \multicolumn{2}{|c|}{ Escola Pública } & \multicolumn{2}{|c|}{ Escola Particular } & \multirow{2}{*}{$p$} \\
\hline & $\mathrm{n}$ & $\%$ & $\mathrm{n}$ & $\%$ & \\
\hline \multicolumn{6}{|l|}{ Escolares } \\
\hline masculino & 73 & 30,7 & 45 & 28,8 & 0,699 \\
\hline feminino & 66 & 20,4 & 21 & 12,4 & 0,027 \\
\hline Pais & 251 & 56,2 & 190 & 65,5 & 0,011 \\
\hline Mães & 175 & 33,7 & 96 & 30,8 & 0,380 \\
\hline
\end{tabular}


Os resultados mostraram que a prevalência de baixo peso foi maior entre os escolares das escolas públicas, independentemente do sexo. Entre os pais dos escolares observou-se uma prevalência de baixo peso, sobrepeso e de obesidade de $0,9 \%$, de $47,4 \%$ e de $12,5 \%$, respectivamente, enquanto entre as mães essas prevalências foram de $2,4 \%, 23,9 \%$ e $8,7 \%$, respectivamente. A prevalência de baixo peso mostrou-se maior nos pais e mães de escolares da rede pública de ensino $(2,2 \%)$ do que naqueles da rede particular $(1,0 \%)$.

Na Tabela 2, comparando-se a prevalência de sobrepeso/obesidade dos pais e mães segundo a rede de ensino dos seus filhos, foi identificado um maior percentual entre os pais das escolas públicas $(65,5 \%)$ em relação àqueles das escolas particulares $(56,2 \%)$, $(p=0,011)$, não havendo diferença estatisticamente significante quanto ao estado antropométrico materno $(p=0,380)$.

A Tabela 3 apresenta a distribuição percentual do estado antropométrico dos escolares, dos pais e das mães investigadas. Os resultados obtidos por meio da razão de prevalência apresentados na Tabela 4 mostram que, quando a mãe tem sobrepeso/obesidade, o escolar do sexo feminino tem uma probabilidade $63 \%$ maior de também apresentá-la $(\mathrm{RP}=1,63$; IC95\%=1,1-3,0; $p=0,02$ ); quando os pais apresentam sobrepeso/obesidade, essa probabilidade no escolar feminino se eleva significantemente para $78 \%$ $(\mathrm{RP}=1,78$; IC95\%=1,1-3,5; $p=0,01)$. Não foi observada relação estatisticamente significante entre o IMC dos pais e mães quando foram considerados escolares do sexo masculino.

\section{Tabela 3}

Distribuição absoluta e percentual do estado antropométrico dos escolares, dos pais e das mães investigados. Florianópolis, SC, 2007.

\begin{tabular}{|c|c|c|c|c|c|c|}
\hline & \multicolumn{2}{|c|}{$\begin{array}{c}\text { Com } \\
\text { sobrepeso/obesidade }\end{array}$} & \multicolumn{2}{|c|}{$\begin{array}{c}\text { Sem } \\
\text { sobrepeso/obesidade }\end{array}$} & \multicolumn{2}{|c|}{ Total } \\
\hline & $\mathrm{n}$ & $\%$ & $\mathrm{n}$ & $\%$ & $n$ & $\%$ \\
\hline \multicolumn{7}{|l|}{ Escolares } \\
\hline masculino & 118 & 29,9 & 276 & 70,1 & 394 & 100,0 \\
\hline feminino & 87 & 17,7 & 405 & 82,3 & 492 & 100,0 \\
\hline Pais & 441 & 59,8 & 737 & 40,2 & 737 & 100,0 \\
\hline Mães & 271 & 32,6 & 560 & 67,4 & 831 & 100,0 \\
\hline
\end{tabular}

Tabela 4

Associação entre o sobrepeso/obesidade nos pais e nos seus filhos, de acordo com o sexo. Florianópolis, SC, 2007.

\begin{tabular}{|c|c|c|c|c|c|}
\hline Variável & Sexo do escolar & Razão de prevalência & IC95\% & $\chi^{2}$ & $p$ \\
\hline Estado & Masculino & 1,22 & $0,8-2,1$ & 0,20 & 0,240 \\
\hline \multicolumn{6}{|c|}{ Antropométrico } \\
\hline da mãe & Feminino & 1,63 & $1,1-3,0$ & 6,10 & 0,020 \\
\hline Estado & Masculino & 1,10 & $0,7-1,8$ & 0,15 & 0,710 \\
\hline \multicolumn{6}{|c|}{ Antropométrico } \\
\hline do pai & Feminino & 1,78 & $1,1-3,5$ & 6,15 & 0,010 \\
\hline
\end{tabular}




\section{Discussão}

Os valores de sobrepeso/obesidade encontrados nos escolares do sexo masculino, de aproximadamente $30 \%$, são semelhantes aos oriundos de estudos em países industrializados, nos quais as prevalências situam-se entre 27 e $33 \%, 26$ enquanto nas meninas estes valores ficaram abaixo da prevalência observada nesses países.

Dados recentes, originados da Pesquisa de Orçamento Familiar (POF) 5 realizada em 2002 pelo Instituto Brasileiro de Geografia e Estatística (IBGE), indicam que a prevalência de sobrepeso incluindo obesidade foi mais elevada em meninos $(17,9 \%)$ do que em meninas $(15,4 \%)$.

Estas prevalências são concordantes com as observadas no presente estudo, onde a ocorrência de sobrepeso/obesidade foi mais elevada em meninos quando comparada à das meninas, o que se assemelha aos resultados encontrados por Marins et al.27 com crianças e adolescentes do Rio de Janeiro. Os nossos resultados indicam também que a prevalência de sobrepeso/obesidade estimada entre os escolares de Florianópolis (29,9\% em meninos e $17,8 \%$ em meninas) é mais elevada do que a encontrada na população brasileira.

Entretanto, os resultados apresentados são discordantes dos relatados por outros autores, que observaram prevalência de sobrepeso/obesidade mais elevada em meninas, 12 ou argumentaram a inexistência de diferenças entre os sexos que possa ser atribuída à condição fisiológica.28,29

No presente estudo o achado de uma prevalência global de sobrepeso incluindo obesidade de $23,1 \%$, semelhante à encontrada em estudo realizado em 2002 no mesmo município, seguindo os mesmos critérios diagnósticos 6 e que identificou um valor de $22,2 \%$ de sobrepeso/obesidade, aponta para uma estabilização na ocorrência destes eventos nesta faixa etária.

Analisando a prevalência de sobrepeso/obesidade segundo a rede de ensino dos escolares, nosso estudo mostrou diferenças para o sexo feminino de ambas as redes de ensino. Porém, ao contrário dos resultados encontrados por Brasil et al., ${ }^{30}$ que registraram prevalência de sobrepeso mais elevada em escolares da rede particular, neste estudo a prevalência de sobrepeso/obesidade foi maior na rede pública de ensino.

Segundo Monteiro e Conde 31 a alta prevalência de sobrepeso/obesidade na população em geral se deve à transição epidemiológica que vem ocorrendo no Brasil, onde o excesso de peso tem aumentado em todos os extratos socioeconômicos, principalmente nas famílias de nível socioeconômico mais baixo. Isso pode acontecer pelo fato de a população de menor poder aquisitivo ter dificuldade em adquirir alimentos mais saudáveis ou ter pouco acesso a informações sobre alimentação e saúde. Nesse sentido, o estudo de Gabriel et al. 32 identificou a ocorrência de hábitos alimentares mais saudáveis entre alunos de escola particular, comparados aos alunos de escola pública, corroborando a idéia de que as famílias de menor nível socioeconômico consomem alimentos mais ricos em gorduras e açúcares simples, possivelmente pelo fácil acesso e baixo preço no mercado, ao contrário dos alimentos mais nutritivos, cujos preços são mais elevados.

A prevalência de baixo peso no presente estudo foi relativamente pequena $(3,9 \%)$ e mostrou-se similar à encontrada em estudo realizado no mesmo município, 6 que apresentou o percentual de 3,7\%. Entretanto, houve aumento da prevalência de sobrepeso comparando-se aos resultados da pesquisa anterior, sugerindo um deslocamento do baixo peso para a escala do sobrepeso/obesidade.

Em relação ao estado antropométrico dos familiares dos escolares, observou-se que a maior prevalência de obesidade identificada nos homens nesse estudo (59,8\%) discorda dos resultados encontrados pela POF 2002-2003 que mostrou ser esta prevalência maior entre as mulheres adultas $(13,1 \%)$ do que entre os homens adultos $(8,9 \%) .5$

Os resultados obtidos em relação à associação entre o IMC dos pais e o dos filhos mostram que a presença de sobrepeso/obesidade nas mães e nos pais influencia o IMC das filhas. As meninas apresentaram prevalência de sobrepeso/obesidade 1,63 vezes maior quando as mães apresentavam sobrepeso ou obesidade e 1,78 vezes maior quando o pai encontrava-se nessa situação, em comparação com mães e pais eutróficos ou de baixo peso. Nossos resultados apresentam semelhanças com vários estudos desenvolvidos no Brasil e em outros países, como o de Burbano et al.,33 que avaliaram os fatores de risco de sobrepeso em meninas de 12 a 19 anos em uma cidade do Equador e observaram correlação direta entre o IMC das participantes e o IMC estimado da mãe. Em outros estudos internacionais, como o de Padez et al. ${ }^{34} \mathrm{em}$ crianças portuguesas de 7 a 9,5 anos, o de Valerio et al. 35 em crianças italianas de sete anos e o de Zeller et al. 36 em jovens de 8 a 16 anos, os valores de IMC dos escolares foram relacionados significantemente aos de seus pais e mães.

É importante destacar que a utilização de medidas antropométricas autorreferidas pelos pais, que é uma técnica de aferição controversa, foi uma 
das limitações desse estudo. Enquanto alguns autores relatam que essa técnica apresenta níveis aceitáveis de validade de informação,22,37 outro estudo aponta que a mesma pode se alterar conforme o sexo ou a idade e também segundo as condições socioeconômicas dos grupos estudados. 38

Também se faz necessário salientar a não avaliação da maturação sexual dos escolares estudados, que pode influenciar na maior ou menor cota de gordura. Porém, a classificação do estado nutricional das crianças e adolescentes, por meio de sua avaliação antropométrica, seguiu as referências do CDC, 19 que estabelece pontos de corte específicos por sexo e idade para o IMC, sendo este método utilizado em outros estudos realizados com a mesma faixa etária e que também não consideram os estágios de maturação sexual dos avaliados. 29

Sabendo-se da dificuldade em tratar a obesidade em indivíduos adultos, principalmente pelo fato de sua associação com outras doenças crônicas não transmissíveis, a prevenção da obesidade iniciada na infância e adolescência parece ser o principal meio de redução da sua prevalência, utilizando-se medidas de intervenção nutricional focadas também no ambiente familiar do escolar, já que o acesso à educação nutricional contribui para a melhoria da qualidade de vida e da saúde nutricional da população. Dessa forma, pode-se considerar que os resultados deste estudo tenham gerado informações que sirvam de subsídios para intervenções na área de política de saúde e nutrição, que visem à prevenção da obesidade em escolares e seus familiares, interagindo com o coti-diano de cada família. Assim poderão ser criados hábitos alimentares mais saudáveis, tornando o ambiente familiar menos propenso ao desenvolvimento dessa doença.

\section{Referências}

1. Fowler-Brown A, Kahwati LC. Prevention and treatment of overweight in children and adolescents. Am Fam Physician. 2004; 69: 2591-8.

2. Oliveira CL, Mello MT, Cintra IP, Fisberg M. Obesidade síndrome metabólica na infância e adolescência. Rev Nutr. 2004; 17: 237-45.

3. Bray GA. Sobrepeso, Mortalidade e Morbidade. In Bouchard C. (Ed.). Atividade física e obesidade. São Paulo: Manole; 2003. p. 35-62.

4. Wang Y, Monteiro C, Popkin BM. Trends of obesity and underweight in older children and adolescents in the United States, Brazil, China, and Russia. Am J Clin Nutr. 2002; 75: 971-7.

5. IBGE (Instituto Brasileiro de Geografia e Estatística) Pesquisa de Orçamentos Familiares 2002-2003. Antropometria e análise do estado nutricional de crianças e adolescentes no Brasil. [2 jun 2007]. Disponível em: http://www.ibge.gov.br/home/estatistica/populacao/ condicaodevida/pof/2003medidas/comentario.pdf

6. Assis MAA, Rolland-Cachera MF, Grosseman S, Vasconcelos FAG, Luna MEP, Calvo MCM, Barros MVG, Pires MMS, Bellisle F. Obesity, overweight and thinness in schoolchildren of the city of Florianópolis, Southern Brazil. Eur J Clin Nutr. 2005; 59: 1015-21.

7. Whitaker RC, Wright JA, Pepe MS, Seidel KD, Dietz WH. Predicting obesity in young adulthood from childhood and parental obesity. N Engl J Med. 1997; 337: 869-73.

8. Cysneiros MAPC. Obesidade na infância e adolescência Pediatr Mod. 1996; 32: 705-16.

9. Escrivão MAMS, Oliveira FLC, Taddei JAAC, Ancona-
Lopez F. Obesidade exógena na infância e na adolescência. J Pediatr. 2000; 76: S305-10.

10. Engstrom EM, Anjos LA. Relação entre o estado nutricional materno e sobrepeso nas crianças brasileiras. Rev Saúde Pública. 1996; 30: 233-9.

11. Giugliano R, Carneiro EC. Fatores associados à obesidade em escolares. J Pediatr. 2004; 80:17-22.

12. Guimarães LV, Barros MBA, Martins MSAS, Duarte EC. Fatores associados ao sobrepeso em escolares. Rev Nutr. 2006; 19: 5-17.

13. Abrantes MM, Lamounier JA, Colosimo EA. Prevalência de sobrepeso e obesidade em crianças e adolescentes das regiões Sudeste e Nordeste. J Pediatr. 2002; 78: 335-40.

14. Salles RK, Kazapi IAM, Di Pietro P. Ocorrência de obesidade em adolescentes da rede de ensino do município de Florianópolis. In: Obesidade e anemia carencial na adolescência: simpósio. São Paulo: Instituto Danone; 2000. $235 \mathrm{p}$.

15. Silva NN. Amostragem probabilística: um curso introdutório. São Paulo: EDUSP; 1998. 124 p.

16. Conde WL, Monteiro CA. Body mass index cutoff points for evaluation of nutritional status in Brazilian children and adolescents. J Pediatr. 2006; 82: 266-72.

17. Lohman TG, Roche AF, Martolell R. Anthropometric standardization reference manual. Illinois: Human Kinetics Books; 1988

18. WHO (World Health Organization). Physical status: the use and interpretation of anthropometry. Geneva; $1995.452 \mathrm{p}$ (Technical Report Series, 854). 
19. CDC/NCHS (Center for Disease Control and Prevention/ National Center for Health Statistics) 2000 CDC Growth Charts: United States. [2 jun 2007]. Disponível em: http://www.cdc.gov/growthcharts

20. Bolton-Smith C, Woodward M, Tunstall-Pedoe H, Morrison C. Accuracy of the estimated prevalence of obesity from self reported height and weight in an adult Scottish population. J Epidemiol Commun Health. 2000; 54: 143-8.

21. Nakamura K, Hoshino Y, Kodama K, Yamamoto M. Reliability of self-reported body height and weight of adult japanese women. J Biosoc Sci. 1999; 31: 555-8.

22. Neto GAB, Polito MD, Lira VA. Fidedignidade entre peso e estatura reportados e medidos e a influência do histórico de atividade física em indivíduos que procuram a prática supervisionada de exercícios. Rev Bras Med Esporte. 2005 11: 141-5.

23. Fonseca MJM, Faerstein E, Chor D, Lopes CS. Validade de peso e estatura informados e índice de massa corporal estudo pró-saúde. Rev Saúde Pública. 2004; 38: 392-8.

24. Chor D, Coutinho ESF, Laurenti R. Reliability of selfreported weight and among state bank employees in Rio de Janeiro. Rev Saúde Pública. 1999; 33: 16-23.

25. Medronho RA. Epidemiologia. São Paulo: Atheneu; 2004.

26. Hanley AJ, Harris SB, Gittelsohn J, Wolever TM, Saksvig B, Zinman B. Overweight amog children and adolescents in a Native Canadian community: prevalence and associated factors. Am J Clin Nutr. 2000; 71: 693-700.

27. Marins VMR, Almeida RM, Pereira RA, Barros MBA Overweight and risk of overweight in schoolchildren in the city of Rio de Janeiro, Brazil: prevalence and characteristics. Ann Trop Paediatrics. 2002; 22: 137-44.

28. Troncon JK, Gomes JP, Guerra-Júnior G, Lalli CA Prevalência de obesidade em crianças de uma escola pública e de um ambulatório geral de Pediatria de hospital universitário. Rev Paul Pediatr. 2007; 25: 305-10.

29. Soar C, Vasconcelos FAG, Assis MAA, Grosseman S, Luna MEP. Prevalência de sobrepeso e obesidade em escolares de uma escola pública de Florianópolis, Santa Catarina. Rev Bras Saúde Matern Infant. 2004; 4: 391-7.

Recebido em 10 de junho de 2008

Versão final apresentada em 30 de novembro de 2009

Aprovado em 4 de dezembro de 2009
30. Brasil LMP, Fisberg M, Maranhão HS. Excesso de peso de escolares em região do Nordeste Brasileiro: contraste entre redes de ensino pública e particular. Rev Bras Saúde Matern Infant. 2007; 7: 405-12.

31. Monteiro CA, Conde WL. Tendência secular da desnutrição e da obesidade na infância na cidade de São Paulo (1974 1996). Rev Saúde Pública. 2000; 34 (Supl): 52-61.

32. Gabriel CG, Santos MV, Vasconcelos FAG. Avaliação de um programa para promoção de hábitos alimentares saudáveis em escolares de Florianópolis, Santa Catarina, Brasil. Rev Bras Saúde Matern Infant. 2008; 8: 299-308.

33. Burbano JC, Fornasini M, Acosta M. Prevalencia y factores de riesgo de sobrepeso em colegialas de 12 a 19 años em una región semiurbana del Ecuador. Rev Panam Salud Publica. 2003; 13: 277-84.

34. Padez C, Mourao I, Moreira P, Rosado V. Prevalence and risk factors for overweight and obesity in Portuguese children. Acta Paediatr. 2005; 94: 1550-7

35. Valerio G, D'Amico O, Adinolfi M, Munciguerra A, D'Amico R, Franzese A. Determinants of weight gain in children from 7 to 10 years. Nutr Metab Cardiovasc Dis. 2006; 16: 272-8.

36. Zeller MH, Reiter-Purtill J, Modi AC, Gutzwiller J, Vannatta K, Davies WH. Controlled Study of Critical Parent and Family Factors in the Obesigenic Environment. Obesity (Silver Spring). 2007; 15: 126-36.

37. Weaver TW, Kushi LH, Mcgovern PG, Potter ID, Rich SS, King RA, Whitbeck J, Greenstein J, Sellers TA. Validation study of self-reported measures of fat distribution. Int $\mathrm{J}$ Obes. 1996; 20: 644-50.

38. Alvarez-Torices JC, Franch-Nadal J, Alvarez-Guisasola F, Hernandez-Mejia R, Cueto-Espina RA. Self-reported height and weight and prevalence of obesity: study in a Spanish population. Int J Obes Relat Metab Disord. 1993; 17: 663- 